\title{
MULTIPOLARIMETRIC SAR IMAGE CHANGE DETECTION BASED ON MULTISCALE FEATURE-LEVEL FUSION
}

\author{
X. Sun*, J. Zhang, L. Zhai \\ Chinese Academy of Surveying and Mapping, 100830 Beijing, China - (sunxx, zhailiang, jxzhang)@ casm.ac.cn
}

Commission VII, WG 6

KEY WORDS: Multipolarimetric SAR, Change Detection, NSCT, Fusion

\begin{abstract}
:
Many methodologies of change detection have been discussed in the literature, but most of them are tested on only optical images or traditional synthetic-aperture radar (SAR) images. Few studies have investigated multipolarimetric SAR image change detection. In this study, we presented a type of multipolarimetric SAR image change detection approach based on nonsubsampled contourlet transform and multiscale feature-level fusion techniques. In this approach, Instead of denoising an image in advance, the nonsubsampled contourlet transform multiscale decomposition was used to reduce the effect of speckle noise by processing only the low-frequency sub-band coefficients of the decomposed image, and the multiscale feature-level fusion technique was employed to integrate the rich information obtained from various polarization images. Because SAR image information is dependent on scale, a multiscale multipolarimetric feature-level fusion strategy is introduced into the change detection to improve change detection precision; this feature-level fusion can not only achieve complementation of information with different polarizations and on different scales, but also has better robustness against noise. Compared with PCA methods, the proposed method constructs better differential images, resulting in higher change detection precision.
\end{abstract}

\section{INTRODUCTION}

The traditional change detection methods were proposed on the basis of stable intensity images such as optical images (Singh 1989; Rignot and van Zyl 1993; Gamba et al. 2006; Geoffrey 2001; Kasetkasem and Varshney 2002; Li 2010; ChaabouniChouayakh et al. 2013; Akiwowo and Eftekhari 2013). However, because of the unique imaging principle of syntheticaperture radar (SAR) images, the radar cross section (RCS) of a scene target characterized by the image has certain randomness and exhibits stochastic distribution. Moreover, it is seriously affected by noise. Therefore, when conventional methods are used to detect SAR image changes, the detection results are generally not ideal.

In multipolarimetric SAR image change detection, several factors must be considered: how to construct the differential image, how to eliminate noise effects, and how to make full use of effective information by using various polarization methods to improve change detection precision. Noise may be reduced by implementing denoising processes (Kharbouche and Clavet 2013) such as multi-look processing and spatial filtering algorithms on the image before changes detection. However, when the noise is removed, some useful change information is also removed because denoising algorithms cause losses of local image information; this is commonly known as the "contradiction between noise removal and detail retention". The nonsubsampled contourlet transform (NSCT) is not only able to compromise on this problem by both suppressing SAR image speckle noise and retaining geometric details, but it can depict SAR image change on various scales. Consequently, this paper applies NSCT in multipolarimetric SAR image change detection and proposes a new type of method of multipolarimetric SAR image change detection based on multiscale feature-level fusion.

\section{METHODS}

To aid understanding of our method, in this section we first introduce the change detection algorithm for single-polarized images in the NSCT domain based on multiscale feature-level fusion. This is the kernel part of the entire algorithm framework (hereafter referred to as the "kernel operator"). We then introduce the change detection method for multipolarimetric SAR images based on this kernel operator.

\subsection{Kernel operator}

The kernel operator of the proposed change detection algorithm is shown in figure 1 .

Its main steps are as follows.

Step 1. Data preprocessing and generation of differential image For single-polarized SAR images $X_{1}$ and $X_{2}$ on time phases $T_{1}$ and $T_{2}$, respectively, conduct geometric correction and registration, then run difference operation to yield a differential image $X_{D}$.

Step 2. For the differential image $X_{D}$, conduct multiscale decomposition and reconstruct an approximated image $X_{D}{ }^{\prime}$ To generate the approximated image, we conducted NSCTbased multiscale decomposition on the differential image $X_{D}$ first to obtain a multiscale series $X_{M S}=\left\{X_{M S}^{0}, \cdots, X_{M S}^{n}, \cdots, X_{M S}^{N-1}\right\} \quad, \quad$ where $n$ 
$(\mathrm{n}=0,1, \ldots, \mathrm{N}-1)$ is the number of decomposition layers. When $n=0$, then $X_{M S}^{0}=X_{D}$, i.e. the differential image before decomposition. $X_{M S}^{n}$ on every scale consists of a lowfrequency image (also referred to as approximate signal) and several high-frequency coefficients, i.e. $X_{M S}^{n}=\left\{C^{n}, D_{1}^{n}, \cdots, D_{j}^{n}, \cdots, D_{J}^{n}\right\} \quad, \quad$ where $C^{n}$ represents the low-frequency coefficient of the $n^{\text {th }}$ decomposition scale image, $D_{j}^{n}$ is the high-frequency subband coefficient of the $n^{\text {th }}$ decomposition scale image in which $\mathrm{j}(\mathrm{j}=0,1, \ldots, \mathrm{J}-1)$ is the number of high-frequency subbands. Because the low-frequency coefficient represents the approximate information of the original image, whereas the high-frequency coefficient contains a large amount of noise, then approximated images on different scales are acquired only by reconstructing the low-frequency image while neglecting high-frequency information. With an increase in the number of decomposition layers $n$, the image features become increasingly ambiguous, and the quantity of information decreases until it is not sufficient to affect the change detection result. Therefore, the most appropriate decomposition scale must be determined. We determined the optimal decomposition scale by using the minimum entropy difference method. After the optimal decomposition scale is determined, the approximated image set for differential images on various scales $X_{D}$ can be acquired.

$$
X_{D}^{\prime}=\left\{X_{D}^{0^{\prime}}, \cdots, X_{D}^{n^{\prime}}, \cdots, X_{D}^{L^{\prime}}\right\}
$$

$L \leq N-1$.

Step 3. Multiscale feature-level fusion and differential image reconstruction

The approximated differential images on different scales contain different difference information, and therefore different change detection results can be extracted from differential images on different scales. Hence, the difference information acquired on different scales can be summarized using the feature-level fusion method so as to reduce differential image dependence on scale. Multiscale fusion is executed on reliable scales only. The multiscale differential images on different scales contain different information quantities: the lower the scale, the greater the quantity of information the corresponding image contains, but the greater the noise component it contains; conversely, the higher the scale, the less information the corresponding image contains, but the lower the noise component it contains. Thus, the differential images on different scales were fused by using the weighted average method after computing the following formula:

$$
X_{F D}=\sum_{n=1}^{L} W^{n} X_{M S}^{n}
$$

where $W^{n}$ represents the weight of the image $X_{M S}^{n}$ on scale $\mathrm{n}$, and its value can be obtained by computing the entropies of the images on various stable scales and normalizing them. $X_{F D}$ is simply the reconstructed differential image.

Step 4. Threshold the reconstructed differential image $X_{F D}$ to generate the change detection result.
An expectation-maximization (EM) algorithm was employed to run iterative operations for the reconstructed differential image $X_{F D}$ to determine the change threshold T. This threshold was then used to split the image and to extract the preliminary change detection result. Usually, abnormal phenomena such as some isolated pixel points or speckle noise will be generated in the preliminary change detection result and must be removed through post-processing. Because a change is often continuous, if a change takes place in the neighbouring pixels of one pixel, then the probability of a change occurring to this pixel would be greater than that of a change not occurring; conversely, if no change takes place in any of its neighbouring pixels, then this pixel is more likely to be unchanged. Consequently, the isolated pixel points or speckle noise can be processed by using greyscale mathematical morphology theory with spatial neighbourhood relationships taken into account, to remedy small vulnerabilities and remove isolated points, leading to the final change detection result $X_{C D}$. For post-processing detection results of tests, we adopted the change detection postprocessing module of ImageInfo software developed by the Chinese Academy of Surveying \& Mapping. The postprocessing was achieved using a scaling algorithm to search the speckle with the maximum sum of pixel numbers within a given window size and to conduct optimal merging.

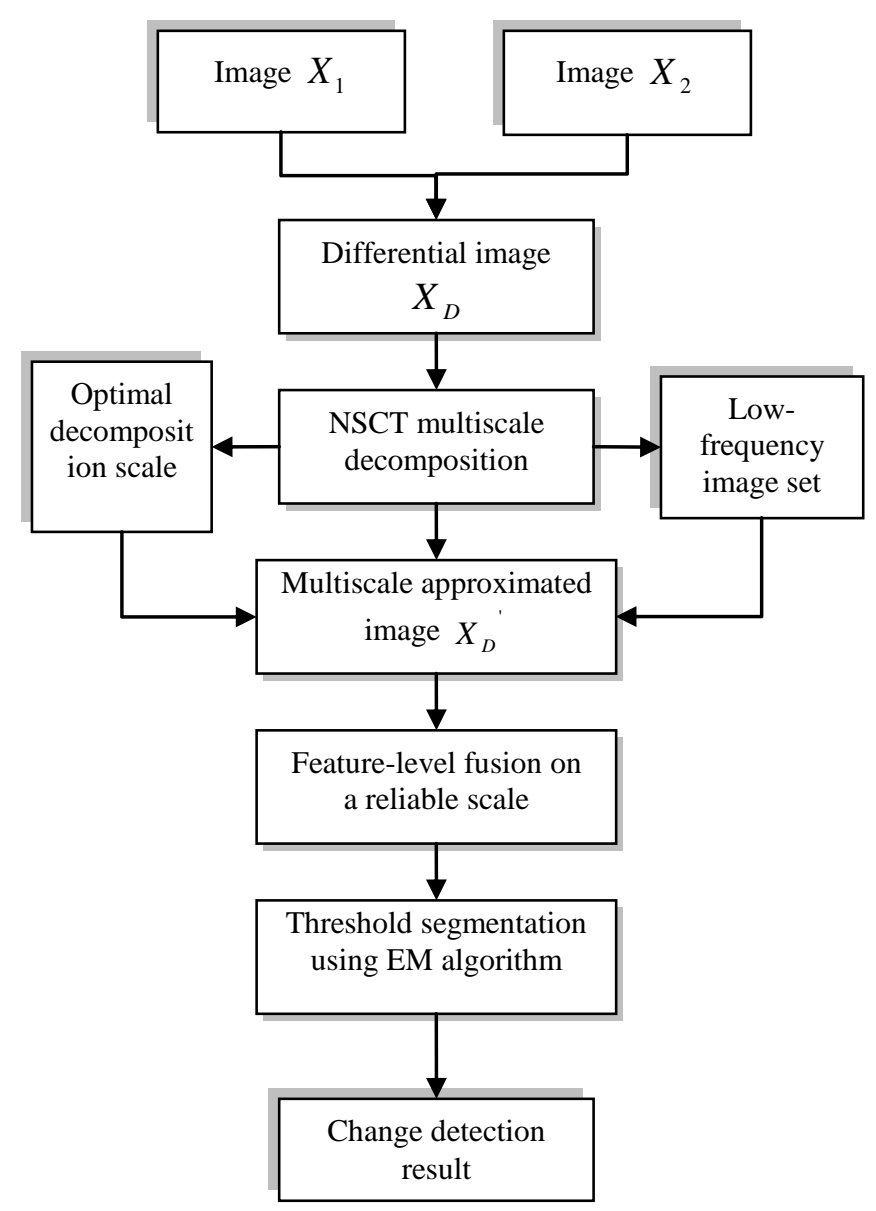

Figure 1. Change detection kernel operator based on multiscale feature-level fusion 


\subsection{Multipolarimetric decision-level fusion}

Let the fully polarized SAR images corresponding to time phases $t_{1}$ and $t_{2}$ be $X^{k}=\left\{X_{H H}^{k}, X_{H V}^{k}, X_{V H}^{k}, X_{V V}^{k}\right\}$, where $k=(1,2)$, which represents the number of time phases. Owing to antenna reciprocity, $\mathrm{HV}=\mathrm{VH}$, only three polarimetric images $X^{k}=\left\{X_{H H}^{k}, X_{H V}^{k}, X_{V V}^{k}\right\}$

were selected for detection.

Different polarimetric images contain different change information. Therefore, all polarimetric images can be used to extract change information respectively, and then decision-level fusion is conducted for the acquired change results. The specific implementation steps are as shown in figure2. First, for every polarimetric image, we implement the kernel operator and obtain the respective change detection results $X_{C D}^{H H}, X_{C D}^{H V}$, and $X_{C D}^{V V}$. Then voting is performed for the spatial pixels of every change detection result (Klein et al. 2008), which is a binary choice for every pixel (change occurring $\omega_{c}$, and change not occurring $\omega_{u}$ ), and the maximal number of ballots $M_{k}(i, j), k=\{u, c\}$ received for the pixel $(i, j)$ is used to discriminate the category of the pixel in the ultimate fusion image, i.e. change category or non-change category. After fusion, the fully polarized change detection result is obtained.

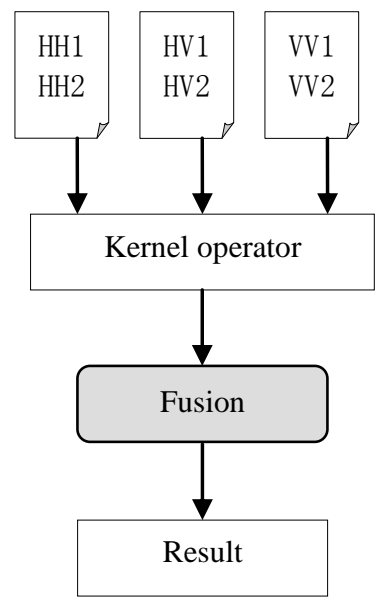

Figure 2 Decision-level fusion on different polarimetric images to detect changes

\section{EXPERIMENTS AND ANALYSIS}

The Japanese ALOS satellite were selected as the experimental data, which were imaged on 5 January 2011 and 7 December 2007. The detailed parameters of the acquired data are shown in table 1, and figure 3(a) and (b) show pseudocolour synthetic images of the polarimetric data at the two time phases.

\begin{tabular}{lll}
\hline Parameter & Time phase 1 & Time phase 2 \\
\hline Date acquired & 20110105 & 20071207 \\
Satellite platform & ALOS & ALOS \\
\hline
\end{tabular}

\begin{tabular}{lll}
\hline Sensor & PALSAR & PALSAR \\
Mode & Fully polarized & Fully polarized \\
Off-nadir angle & 21.5 & 21.5 \\
Sampling resolution & $12.5 \mathrm{~m}$ & $12.5 \mathrm{~m}$ \\
Product format & CEOS & CEOS \\
Number of bits & 16 & 16 \\
\hline
\end{tabular}

Table 1. Image acquisition properties.

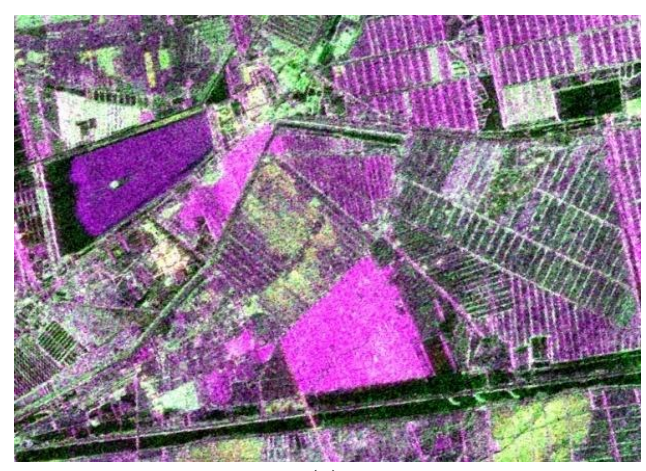

(a)

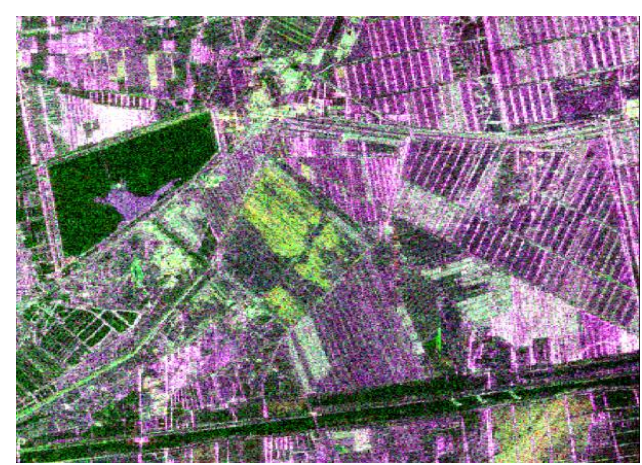

(b)

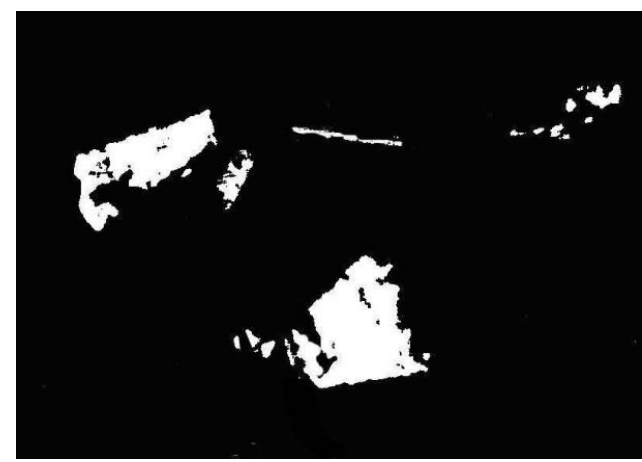

(c)

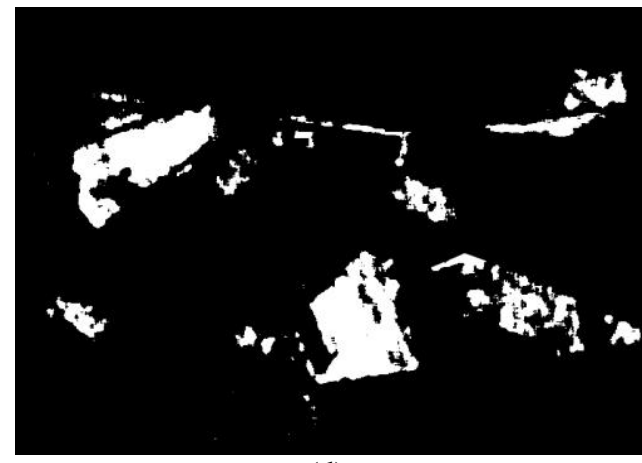

(d) 
Figure 3 Change detection using the proposed method and PCA separately

(a) 2007 PALSAR fully polarized image, (b) 2011 PALSAR fully polarized image, (c) Change detection using the proposed method, and (d) Change detection using the PCA

To verify the effectiveness of the proposed approach, a change detection test using PCA was performed for the same data. The test result is shown in figure $3(\mathrm{c})$ and $3(\mathrm{~d})$. By comparing the detection results obtained by PCA and those obtained by our proposed approach, we can see that the PCA detection result contains a large amount of false alarm information and is poor in extracting the change of linear features because it exhibits a large amount of omission. To quantitatively compare the detection performance of the two fusion methods, we obtained statistics of the missed detection rate and false alarm rate, as shown in table 2 . When the missed detection rates are relatively close to each other, the false alarm rate of our proposed approach is clearly lower than that of PCA. The statistics further verify that the PCA detection result contains more false change information than the result of our proposed approach. Comparison of the detection results indicates that the missed detection rate obtained by our proposed approach mainly arises from the difference between the speckle area we detected by automatic methods and the change area interpreted manually. This is mainly due to the addition of boundary judgment from a priori knowledge when manually drawing the reference change image, whereas the change detection in an automatic detection method is based only on the features manifested by the image itself.

\begin{tabular}{lll}
\hline Detection method & $\begin{array}{l}\text { Missed } \\
\text { detection } \\
\text { rate }\end{array}$ & False alarm rate \\
\hline PCA & $13.70 \%$ & $20.51 \%$ \\
$\begin{array}{l}\text { Our proposed } \\
\text { method }\end{array}$ & $12.89 \%$ & $8.93 \%$ \\
\hline
\end{tabular}

Table 2. Comparison of the detection performance of the proposed method and that of PCA

\section{CONCLUSION}

Change detection is one important application of multipolarimetric SAR images. However, because multipolarimetric SAR images have complex electromagnetic properties and characteristics such as serious susceptibility to noise, change detection in multipolarimetric SAR images is difficult. In this paper, we proposed a type of new multipolarimetric SAR image change detection algorithm in the NSCT domain based on multiscale feature-level fusion, primarily targeting the features of the multipolarimetric SAR image itself and the existing problems of change detection. This algorithm reduces the effect of speckle noise by processing lowfrequency sub-band coefficients for NSCT multiscale decomposition. We introduced a multiscale feature-level fusion strategy into the change detection algorithm, which then fused and improved the information with different polarizations and on different scales relatively well.

\section{ACKNOWLEDGEMENTS}

This research was supported by National Natural Science Foundation of China (grant number 41371406), Open Research Fund of Key Laboratory of GeoInformatics of National Administration of Surveying, Mapping and Geoinformation (7771514 and 77142104), and Basic Research Fund of Chinese Academy of Surveying and Mapping (7771507 and 7771509).

\section{REFERENCES}

Akiwowo, A. and Eftekhari, M., 2013, Feature-based detection using Bayesian data fusion. International Journal of Image and Data Fusion, 4(4), pp. 308-323.

Chaabouni-Chouayakh, H., Arnau, I.R. and Reinartz, P., 2013, Towards automatic 3-D change detection through multi-spectral and digital elevation model information fusion. International Journal of Image and Data Fusion, 4(1), pp. 89-101.

Gamba, P., Dell'Acqua, F. and Lisini, G., 2006, Change detection of multitemporal SAR data in urban areas combining feature-based and pixel-based techniques. IEEE Transactions on Geoscience and Remote Sensing, 44(10), pp. 2820-2827.

Geoffrey, G.H., 2001, Object-level change detection in spectral imagery. IEEE Transactions on Geoscience and Remote Sensing, 39(3), pp. 553-561.

Kasetkasem, T. and Varshney, P., 2002, An image change detection algorithm based on Markov random field models. IEEE Transactions on Geoscience and Remote Sensing, 40(4), pp. 1815-1823.

Kharbouche, S. and Clavet, D., 2013, Speckle reducing in PolSAR images for topographic feature extraction. International Journal of Image and Data Fusion, 4(2), pp. 146158.

Li, D.R., 2010, Remotely sensed images and GIS data fusion for automatic change detection. International Journal of Image and Data Fusion, 1(1), pp. 99-108.

Rignot, E.J.M. and van Zyl, J.J, 1993, Change detection techniques for ERS-1 SAR data. IEEE Transactions on Geoscience and Remote Sensing, 31(4), pp. 896-906.

Singh, A., 1989, Digital change detection techniques using remotely sensed data. International Journal of Remote Sensing, 10(6), pp. 989-1003.

Sun, X.X., Zhang, J.X., Yan, Q. and Gao, J.X, 2010, Fusion of multiple polarimetric SAR images using nonsubsampled contourlet transform. 2010 International Conference on Information Security and Artificial Intelligence, ISAI 2010, pp. V3-535-538. 